\title{
Search for rare processes with DAMA/LXe experiment at Gran Sasso
}

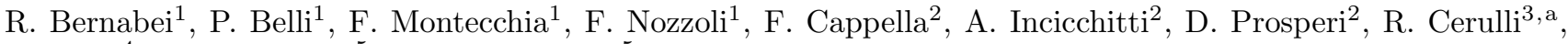 \\ C.J. Dai ${ }^{4}$, V.Yu. Denisov ${ }^{5}$, and V.I. Tretyak ${ }^{5}$ \\ 1 Dipartimento di Fisica, Università di Roma "Tor Vergata" and INFN, Sezione di Roma II, I-00133, Roma, Italy \\ 2 Dipartimento di Fisica, Università di Roma "La Sapienza" and INFN, Sezione di Roma, I-00185, Roma, Italy \\ 3 INFN - Laboratori Nazionali del Gran Sasso, I-67010 Assergi (AQ), Italy \\ 4 IHEP, Chinese Academy, P.O. Box 918/3, Beijing 100039, PRC \\ ${ }^{5}$ Institute for Nuclear Research, MSP 03680 Kiev, Ukraine
}

Received: 20 June 2005 /

Published online: 22 February 2006 - C Società Italiana di Fisica / Springer-Verlag 2006

\begin{abstract}
After a short introduction on the low background liquid xenon DAMA set-up (DAMA/LXe) and its main previous results, we discuss the search for the nucleon, di-nucleon and tri-nucleon decays into invisible channels (disappearance or decay to neutrinos, Majorons, etc.) in the ${ }^{136}$ Xe isotope. The obtained limits $\left(90 \%\right.$ C.L.) on the lifetimes are: $\tau_{n}>3.3 \cdot 10^{23} \mathrm{yr}, \tau_{p}>4.5 \cdot 10^{23} \mathrm{yr}, \tau_{n p}>3.2 \cdot 10^{23} \mathrm{yr}$, $\tau_{p p}>1.9 \cdot 10^{24} \mathrm{yr}, \tau_{n n p}>1.4 \cdot 10^{22} \mathrm{yr}, \tau_{n p p}>2.7 \cdot 10^{22} \mathrm{yr}$ and $\tau_{p p p}>3.6 \cdot 10^{22} \mathrm{yr}$. In particular, the tri-nucleon decay into invisible channels is investigated here for the first time.
\end{abstract}

PACS. 29.40.Mc Scintillation detectors - 95.35.+d Dark matter (stellar, interstellar, galactic, and cosmological) - 11.30.Fs Global symmetries (e.g., baryon number, lepton number)

\section{Introduction on previous results}

The DAMA/LXe experiment has realized several prototype detectors and, then, has preliminarily put in measurement the set-up used in the data taking of refs. [1,2]; this set-up was firstly upgraded in fall 1995 as mentioned in refs. $[3,4,5,6,7]$. In particular, it has an inner vessel filled by $\simeq 6.5 \mathrm{~kg}$ (i.e. $\simeq 2 \mathrm{l}$ ) of liquid xenon. Firstly it used Kr-free xenon enriched in ${ }^{129}$ Xe at $99.5 \%$ [8]; then, in 2000 the set-up was deeply modified reaching the configuration in fig. 5 of ref. [8] to handle also $\mathrm{Kr}$-free xenon enriched in ${ }^{136} \mathrm{Xe}$ at $68.8 \%[9,10]$. In this latter case, the interest has mainly been focused on the higher energy region. The main features of the set-up, details on the data acquisition, on the cryogenic and vacuum systems and on the running parameters control are described in $[6,7,8,10,11]$. In particular, the energy scale in the high energy region is determined with the help of external standard gamma sources, having identified the possibility to perform high energy calibrations with external sources near a flange located on the top of the detector, opening a limited upper part of the external shield. The energy resolution for ${ }^{208} \mathrm{Tl}$ line $\left(E_{\gamma}=2614 \mathrm{keV}\right)$ is $\sigma \simeq 220 \mathrm{keV}$, while for ${ }^{137} \mathrm{Cs}$ line $\left(E_{\gamma}=662 \mathrm{keV}\right)$, which is the source usually exploited for the routine calibrations, $\sigma$ is $\simeq 70 \mathrm{keV}[10,11,7]$.

\footnotetext{
a e-mail: riccardo.cerulli@lngs.infn.it
}

We pointed out the interest in using liquid xenon as target-detector for particle Dark Matter (DM) investigation deep underground since long time [12]. After preliminary measurements both on elastic and inelastic DM particles- ${ }^{129} \mathrm{Xe}$ scattering $[1,3]$, the recoil/electron light ratio and pulse shape discrimination capability in a similar pure LXe scintillator have been measured both with AmB neutron source and with $14 \mathrm{MeV}$ ENEA-Frascati neutron generator [6]. Moreover, in 2000/2001 further measurements on the recoil/electron light ratio with $2.5 \mathrm{MeV}$ ENEA-Frascati neutron generator have also been carried out; see ref. [5] for details and comparisons.

After upgrading of the LXe set-up, new results on the DM particles investigation have been obtained $[4,6]$. In particular, in ref. [6] pulse shape discrimination between recoils and electromagnetic background in the developed pure LXe scintillators has been exploited. Afterwards the inelastic excitation of ${ }^{129} \mathrm{Xe}$ by DM particles with spin-dependent coupling has further been investigated in ref. [4].

Several other rare processes have also been searched for by means of the detector filled with the Kr-free xenon gas enriched in ${ }^{129} \mathrm{Xe}$. In particular, as regards the electron stability, limits on the lifetime of the electron decay in both the disappearance and the $\nu_{e}+\gamma$ channels were set in ref. [2]. The latter has been more recently improved up to $2.0(3.4) \cdot 10^{26}$ yr at $90 \%(68 \%)$ C.L. [13]. Furthermore, 
new lifetime limits on the charge non-conserving electron capture with excitation of ${ }^{129} \mathrm{Xe}$ nuclear levels have also been established to be in the range (1-4) $\cdot 10^{24}$ yr at $90 \%$ C.L. for the different excited levels of ${ }^{129} \mathrm{Xe}$ [14]. The stringent restrictions on the relative strengths of charge nonconserving $(\mathrm{CNC})$ processes have been consequently derived: $\epsilon_{W}^{2}<2.2 \cdot 10^{-26}$ and $\epsilon_{\gamma}^{2}<1.3 \cdot 10^{-42}$ at $90 \%$ C.L. [14].

Moreover, we have searched for the nucleon and dinucleon decay into invisible channels in the ${ }^{129} \mathrm{Xe}$ isotope by exploiting a new approach [15]. It consisted in a search (in the real-time experiment) for radioactive decay of unstable daughter nuclei which were created in result of the $N$ or $N N$ disappearance in parent nucleus. If the half-life of the daughter nucleus is of the order of $1 \mathrm{~s}$ or greater, such a decay will be time-resolved from prompt products if they were emitted and observed in a detector. This approach has the advantage of a branching ratio close to 1 and - if the parent and daughter nuclei are located in the detector itself- also of an efficiency close to 1 . The obtained limits at $90 \%$ C.L. are: $\tau(p \rightarrow$ invisible channels $)>$ $1.9 \cdot 10^{24} \mathrm{yr} ; \tau(p p \rightarrow$ invisible channels $)>5.5 \cdot 10^{23} \mathrm{yr}$ and $\tau(n n \rightarrow$ invisible channels $)>1.2 \cdot 10^{25} \mathrm{yr}$. These limits are similar to or better than those previously available; moreover, they are valid for every possible disappearance channel [15] and the limits for the di-nucleon decay in $\nu_{\tau} \bar{\nu}_{\tau}$ are set there for the first time.

Afterwards, measurements have been carried out by using the $\mathrm{Kr}$-free xenon gas containing $17.1 \%$ of ${ }^{134} \mathrm{Xe}$ and $68.8 \%$ of ${ }^{136} \mathrm{Xe}$ to investigate the ${ }^{134} \mathrm{Xe}$ and ${ }^{136} \mathrm{Xe}$ double beta decay modes. After the preliminary results of refs. $[9$ $16]$ a joint analysis of the $0 \nu \beta \beta$ decay mode in ${ }^{134} \mathrm{Xe}$ and in ${ }^{136} \mathrm{Xe}$ (as suggested in ref. [17]) has been carried out. New lower limits on various $\beta \beta$ decay modes have been obtained: for the $0 \nu \beta \beta\left(0^{+} \rightarrow 0^{+}\right)$decay mode in ${ }^{134} \mathrm{Xe}$ and in ${ }^{136} \mathrm{Xe}$ the limits at $90 \%$ C.L. are $T_{1 / 2}=5.8 \cdot 10^{22} \mathrm{yr}$ and $T_{1 / 2}=1.2 \cdot 10^{24} \mathrm{yr}$, respectively; they correspond to a limit value on effective light Majorana neutrino mass ranging from $1.1 \mathrm{eV}$ to $2.9 \mathrm{eV}$ (90\% C.L.), depending on the adopted theoretical model. For the neutrinoless double beta decay with Majoron $(M)$ in the ${ }^{136} \mathrm{Xe}$ isotope the limit is $T_{1 / 2}=5.0 \cdot 10^{23} \mathrm{yr}(90 \%$ C.L. $)$; for the $2 \nu \beta \beta\left(0^{+} \rightarrow 0^{+}\right)$and the $2 \nu \beta \beta\left(0^{+} \rightarrow 2^{+}\right)$decay modes in ${ }^{136} \mathrm{Xe}$ the limits at $90 \%$ C.L. are $1.0 \cdot 10^{22} \mathrm{yr}$ and $9.4 \cdot 10^{21} \mathrm{yr}$, respectively. It is worthy to note that the experimental limit on the $2 \nu \beta \beta\left(0^{+} \rightarrow 0^{+}\right)$decay mode is in the range of the theoretical estimate by [18] $\left(2.11 \cdot 10^{22} \mathrm{yr}\right)$ and about a factor 5 higher than that of ref. $[19]^{1}$.

A search for the charge non-conserving decay of ${ }^{136} \mathrm{Xe}$ into ${ }^{136} \mathrm{Cs}$ has also been performed for the first time [7], using the data collected during $8823.54 \mathrm{~h}$ and already published in ref. [10]. The used approach has been the investigation of the CNC processes by the search for the possible CNC decay firstly considered in [20]: if in a $\beta$ decay $(A, Z) \rightarrow(A, Z+1)+e^{-}+\bar{\nu}_{e}$ some massless uncharged particle would be emitted instead of the electron (e.g., $\nu_{e}$ or $\gamma$ or Majoron), an additional $511 \mathrm{keV}$ energy

\footnotetext{
1 On the other hand, similar theoretical estimates suffer from the large uncertainties typically associated to the calculations of the nuclear matrix elements.
}

release would occur. Thus, usually forbidden decays to the ground state or to the excited levels of the daughter nuclei would become energetically possible. The presence of the $(A, Z+1)$ isotope or of its daughter products in a sample, initially free from them, would indicate the existence of the CNC decay searched for. Large advantages arise when the so-called "active-source" technique ( source $=$ detector) is considered as in the case described here. In particular, after the possible ${ }^{136} \mathrm{Xe}$ CNC decay, the daughter nucleus ${ }^{136} \mathrm{Cs}$ will be created. It is $\beta$ unstable $\left(T_{1 / 2}=13.16 \mathrm{~d}\right)$ with quite high energy release $\left(Q_{\beta}=2.548 \mathrm{MeV}\right)$ [7]. Comparing the experimental energy distribution with the expected response function, no evidence for the effect searched for has been found. Thus, the lifetime limit is: $\tau_{C N C}\left({ }^{136} \mathrm{Xe} \rightarrow{ }^{136} \mathrm{Cs}\right)>1.3 \cdot 10^{23} \mathrm{yr}$ at $90 \%$ C.L. This limit is one of the highest available limits for similar processes [7]; however, the bound on the charge non-conserving admixture in the weak interactions, which can be derived according to ref. [21], is modest mainly due to the big change in nuclear spin in the considered $\mathrm{CNC}$ transition $\left(\Delta J^{\Delta \pi}=5^{+}\right)$.

\section{The search for nucleon instabilities into invisible channels in the ${ }^{136} \mathrm{Xe}$ isotope}

The baryon number $(B)$ conservation was introduced by Stuckelberg [22] and Wigner [23] more than fifty years ago and remains an accidental symmetry of the Standard Model, when this is seen as a renormalizable theory, not explained by deeper theoretical understanding. Modern theories of particle physics (GUTs, SUSY), unifying quarks and leptons into the same multiplets and predicting new interactions which transform quarks into leptons, naturally lead to the decay of the protons and the otherwise stable bound neutrons [24]. Many decay mechanisms, which violate $B$ on one or two units, have been discussed [24,25]. In a recent work [26], a new process was examined in which two neutrons simultaneously decay to bulk Majoron, $n n \rightarrow M$, with typical lifetime $10^{32}$ $10^{39} \mathrm{yr}$; due to weak coupling of Majorons to normal matter they are not detected in an experiment, and such a process looks as a disappearance of two neutrons from a nucleus. Also mechanisms for the tri-nucleon decay have been proposed in the literature; in particular, very recently in ref. [27] also tri-nucleon decay processes with $\Delta B=3$ have been considered. Moreover, disappearance of particles (electrons, $e^{-}$, or nucleons, $N$ ) are expected also in theories with extra dimensions $[28,29,30]$. No processes with baryon number violation were detected to date. We refer to our previous article [15] for a review of various ideas used in the search for the $N$ and $N N$ decays into invisible channels.

Here a search for the $N, N N$ and $N N N$ instabilities in the ${ }^{136} \mathrm{Xe}$ isotope is described, looking for decays of the unstable daughter nuclei. The experimental energy distribution collected during $8823.54 \mathrm{~h}$ by the LXe scintillator (enriched in ${ }^{136} \mathrm{Xe}$ at $68.8 \%$ ) in the energy region 550 $3550 \mathrm{keV}$ (the same as in refs. [10] and [7]) is shown later. 
Table 1. Daughter nuclei produced in $N, N N$ and $N N N$ decays in ${ }^{136} \mathrm{Xe}$ when the de-excitation of the daughter nucleus occurs by $\gamma$ emission. The half-life times of the isotopes involved in the decay chains vary from $2.5 \mathrm{~m}\left({ }^{133} \mathrm{Sb}\right)$ to $5.243 \mathrm{~d}$ $\left({ }^{133} \mathrm{Xe}\right)$ assuring that the chains are in equilibrium and that subsequent decays are well separated in time.

\begin{tabular}{|c|c|c|}
\hline$\overline{\text { Decay }}$ & $\begin{array}{l}\text { Daughter } \\
\text { nucleus }\end{array}$ & $\begin{array}{l}\text { Subsequent } \\
\text { decays }\end{array}$ \\
\hline$n$ & ${ }^{135} \mathrm{Xe}$ & ${ }^{135} \mathrm{Xe} \stackrel{\beta^{-}}{\longrightarrow}{ }^{135} \mathrm{Cs}{ }^{*}$ \\
\hline$p$ & ${ }^{135} \mathrm{I}$ & ${ }^{135} \mathrm{I} \stackrel{\beta^{-}}{\longrightarrow}{ }^{135} \mathrm{Xe} \stackrel{\beta^{-}}{\longrightarrow}{ }^{135} \mathrm{Cs}^{*}$ \\
\hline$n n$ & ${ }^{134} \mathrm{Xe}$ & Stable \\
\hline$n p$ & ${ }^{134} \mathrm{I}$ & ${ }^{134} \mathrm{I} \stackrel{\beta^{-}}{\longrightarrow}{ }^{134} \mathrm{Xe}$ \\
\hline$p p$ & ${ }^{134} \mathrm{Te}$ & ${ }^{134} \mathrm{Te} \stackrel{\beta^{-}}{\longrightarrow}{ }^{134} \mathrm{I} \stackrel{\beta^{-}}{\longrightarrow}{ }^{134} \mathrm{Xe}$ \\
\hline$n n n$ & ${ }^{133} \mathrm{Xe}$ & ${ }^{133} \mathrm{Xe} \stackrel{\beta^{-}}{\longrightarrow}{ }^{133} \mathrm{Cs}$ \\
\hline$n n p$ & ${ }^{133} \mathrm{I}$ & ${ }^{133} \mathrm{I} \stackrel{\beta^{-}}{\longrightarrow}{ }^{133} \mathrm{Xe} \stackrel{\beta^{-}}{\longrightarrow}{ }^{133} \mathrm{Cs}$ \\
\hline$n p p$ & ${ }^{133} \mathrm{Te}$ & ${ }^{133} \mathrm{Te} \stackrel{\beta^{-}}{\longrightarrow}{ }^{133} \mathrm{I} \stackrel{\beta^{-}}{\longrightarrow}{ }^{133} \mathrm{Xe} \stackrel{\beta^{-}}{\longrightarrow}{ }^{133} \mathrm{Cs}$ \\
\hline$p p p$ & ${ }^{133} \mathrm{Sb}$ & ${ }^{133} \mathrm{Sb} \stackrel{\beta^{-}}{\longrightarrow}{ }^{133} \mathrm{Te} \stackrel{\beta^{-}}{\longrightarrow}{ }^{133} \mathrm{I} \stackrel{\beta^{-}}{\longrightarrow}{ }^{133} \mathrm{Xe} \stackrel{\beta^{-}}{\longrightarrow}{ }^{133} \mathrm{Cs} \quad{ }^{* *}$ \\
\hline
\end{tabular}

* ${ }^{135} \mathrm{Cs}$ is not stable, but has $T_{1 / 2}=2.3 \cdot 10^{6} \mathrm{yr}$ and breaks the decay chain.

** Given here only the main part of the chain.

We remind that a cut has been applied to reject events whose charge is dominant in one PMT, that is to reject - as much as possible - background contribution from outside the inner vessel. As a consequence, a correction (which depends on the used hardware cut in high energy measurements) estimated by Monte Carlo program, properly considering the real geometry and the features of the detector, has been applied to the rate.

The isotopes given in table 1 are produced [31] after the disappearance of one, two or three nucleons in the parent ${ }_{54}^{136} \mathrm{Xe}$ nucleus, when the daughter de-excitation occurs only by $\gamma$ emission. In general, the created daughter nucleus - with one, two or three holes in nuclear shells due to disappeared nucleons - will be in an excited state, unless the nucleons were on the outermost shell. The holes will be filled in the subsequent de-excitation process in which different particles could be emitted. If the excitation energy is lower than the binding energy of the least bound nucleon, only $\gamma$ quanta will be emitted; otherwise, heavy particles such as $p, n$ or $\alpha$ will be ejected leading to daughter nuclei with lower atomic masses and numbers. In that follows we will take into account the $N, N N$ and $N N N$ disappearance from a few outermost shells in the parent nucleus, when only $\gamma$ 's would be emitted, avoiding in this way uncertainty in created daughter nuclide.

The lifetimes for the $N, N N$ and $N N N$ disappearance in ${ }^{136} \mathrm{Xe}$ can be calculated using the formula:

$$
\tau=\frac{\epsilon_{\Delta E} \cdot N_{\text {nucl }} \cdot N_{\mathrm{obj}}^{\mathrm{eff}} \cdot T}{S_{\Delta E}}
$$

where $N_{\text {nucl }}=2.00 \cdot 10^{25}$ is the number of ${ }^{136} \mathrm{Xe}$ nuclei; $T=8823.54 \mathrm{~h}$ is the time of measurement; $N_{\mathrm{obj}}^{\mathrm{eff}}$ is the "effective" number of objects $(n, p, N N$ pairs or $N N N$
Table 2. Values of $N_{\mathrm{obj}}^{\mathrm{eff}}$ used in the present data analysis to search for $N, N N$ and $N N N$ disappearance in ${ }^{136} \mathrm{Xe}$. See ref. [15] and text.

\begin{tabular}{lcccccccc}
\hline Decay & $n$ & $p$ & $n p$ & $p p$ & $n n n$ & $n n p$ & $n p p$ & $p p p$ \\
\hline$N_{\mathrm{obj}}^{\mathrm{eff}}$ & 32 & 26 & 2 & 7 & 1 & 1 & 1 & 1 \\
\hline \hline
\end{tabular}

groups) whose disappearance in the parent nucleus will result in the creation of the specific daughter nuclide; $S_{\Delta E}$ is the number of events which can be ascribed to the decay process searched for in the considered energy window, $\Delta E$, while $\epsilon_{\Delta E}$ is the related detection efficiency.

Equation (1) requires the knowledge of the number of objects, $N_{\text {obj }}^{\text {eff }}$, that was calculated following the method already used in ref. [15]. As regards the $N N N$ decays into invisible channels, there is no available information from previous search and, in particular, there are no reference criteria to evaluate $N_{\mathrm{obj}}^{\mathrm{eff}}$ in these cases. Thus, in the search we have carried out, we cautiously assumed $N_{\mathrm{obj}}^{\mathrm{eff}}=1$ for all the $N N N$ processes $(n n n, n n p, n p p$ and $p p p)$, as done in the search for the $N N$ decays into invisible channels in ref. [32]. The used values of $N_{\mathrm{obj}}^{\mathrm{eff}}$ are summarized in table 2 . Referring to table 1 , we note that, except for the ${ }^{134} \mathrm{Xe}$ nucleus which is stable and does not allow us to search for the $n n$ disappearance, all other daughter nuclei are radioactive. In the subsequent decays (third column of table 1 ), if we exclude the ${ }^{135} \mathrm{Cs}$ which has $T_{1 / 2}=2.3 \cdot 10^{6} \mathrm{yr}$ and breaks the decay chain, the half-lives of the involved nuclides are relatively small. This ensures equilibrium in chains and thus equal number of decays for ${ }^{135} \mathrm{I}$ and ${ }^{135} \mathrm{Xe}$ (in case of the $p$ disappearance), for ${ }^{134} \mathrm{Te}$ and ${ }^{134} \mathrm{I}$ ( $p p$ decay), etc. The expected response functions of the LXe detector for the $\beta^{-}$decays of the nuclei involved in the decay chains were simulated with the help of the EGS4 package [33]. The whole schemes of the decays, using the information from ref. [34] for the $A=133$ isotopes, from ref. [35] for the $A=134$ isotopes and from ref. [36] for the $A=135$ isotopes, were implemented in an event generator which described the initial kinematics of the events. The response functions for the $N, N N$ and $N N N$ disappearance are given by a linear combination of the response functions obtained for the single decays of the generated decay chain. Comparison of the experimental spectrum with the calculated response functions gives no strong indication of the signals searched for. Thus, we limit ourselves to extract only the limits on the probability of these processes. We will see that, except for the $n p$ and $p p$ channel, the calculated response function do not present any distinctive structure, as a peak, at energies above the experimental energy threshold $(550 \mathrm{keV})$ but their shapes are similar to some extent to that of the measured spectrum. As a consequence, the limit on the amplitude of the expected response function, which can be hidden in the experimental data, was determined in a very cautious and simple way (used also in the investigation of other rare processes). In fact, it has been required that - in no energy interval, $\Delta E$ - the number of events which could be ascribed to the investigated process, $S_{\Delta E}$, 

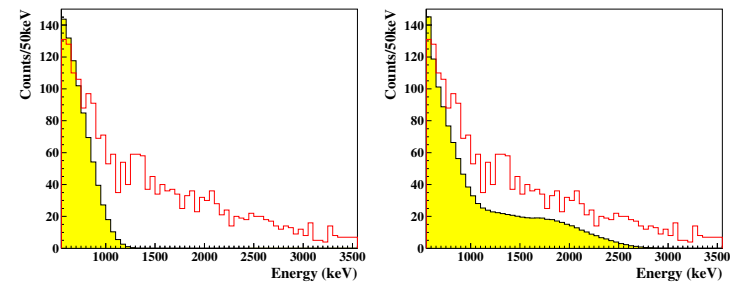

Fig. 1. Comparison between the experimental spectrum measured during $8823.54 \mathrm{~h}$ (thick histogram) and the expected signal (colored histogram) for: left) the $n$ disappearance with $\tau_{n}=3.3 \cdot 10^{23} \mathrm{yr}$ excluded at $90 \%$ C.L.; right) the $p$ disappearance with $\tau_{p}=4.5 \cdot 10^{23} \mathrm{yr}$ excluded at $90 \%$ C.L.

can exceed the number of measured events plus $m \times \sigma$ (where $m$ determines the C.L.). It is evident that the derived limits are very conservative because at least some events in the experimental spectrum can be induced by residual radioactive contaminations.

\subsection{Results for $\mathbf{N}$ decays into invisible channel}

A possible $n$ disappearance in the ${ }^{136} \mathrm{Xe}$ nucleus is followed by the $\beta^{-}$decay ${ }^{135} \mathrm{Xe} \rightarrow{ }^{135} \mathrm{Cs}\left(T_{1 / 2}=9.14 \mathrm{~h}\right.$, $\left.Q_{\beta^{-}}=1151 \mathrm{keV}\right)$ in the sensitive volume of DAMA/LXe. The expected energy distribution is mainly determined by the $\beta^{-}$decay with end-point $\sim 900 \mathrm{keV}$ (96\% of the cases) followed by a $250 \mathrm{keV}$ de-excitation $\gamma$ (the $\beta^{-}$energy distribution is moved towards a higher energy of $250 \mathrm{keV}$ ) [11]. In the data analysis we considered the 550 $700 \mathrm{keV}$ energy region of the experimental spectrum (see fig. 1 left), where 369 events were accumulated. It gives $S_{\Delta E}<369+1.29 \cdot \sqrt{369}=393.6$ at $90 \%$ C.L. The related efficiency, calculated with the EGS4, is $\varepsilon_{\Delta E}=20.2 \%$. Substituting these values into eq. (1) together with the effective number of neutrons which should be taken into account $\left(N_{n}^{\text {eff }}=32\right)$, we obtain the following restriction on the neutron lifetime:

$$
\tau_{n}>3.3 \cdot 10^{23} \mathrm{yr} \text { at } 90 \% \text { C.L. }
$$

The $p$ disappearance in the ${ }^{136} \mathrm{Xe}$ nucleus is instead followed by a chain of two $\beta^{-}$decays: ${ }^{135} \mathrm{I} \rightarrow{ }^{135} \mathrm{Xe}$ $\left(T_{1 / 2}=6.57 \mathrm{~h}, Q_{\beta^{-}}=2648 \mathrm{keV}\right)$ and ${ }^{135} \mathrm{Xe} \rightarrow{ }^{135} \mathrm{Cs}$ (see above). The expected energy distribution for the ${ }^{135} \mathrm{I} \beta^{-}$ decay is characterized by a peak due to the $\sim 527 \mathrm{keV}$ metastable state of ${ }^{135} \mathrm{Xe}\left(T_{1 / 2}=15.29 \mathrm{~m}\right)$, but below the energy threshold considered in this data analysis; the response function for the $p$ disappearance is given by the ${ }^{135} \mathrm{I}+{ }^{135} \mathrm{Xe}$ distribution [11]. The most sensitive energy region of the experimental spectrum is $550-600 \mathrm{keV}$ (see fig. 1 right). For 131 accumulated events, the 90\% C.L. limit is $S_{\Delta E}<145.7$; taking into account the calculated efficiency $\varepsilon_{\Delta E}=12.7 \%$ to detect the ${ }^{135} \mathrm{I}+{ }^{135} \mathrm{Xe}$ decays in the $550-600 \mathrm{keV}$ interval and the effective number of protons $N_{p}^{\mathrm{eff}}=26$, the corresponding limit for the proton lifetime is:

$$
\tau_{p}>4.5 \cdot 10^{23} \text { yr at } 90 \% \text { C.L. }
$$
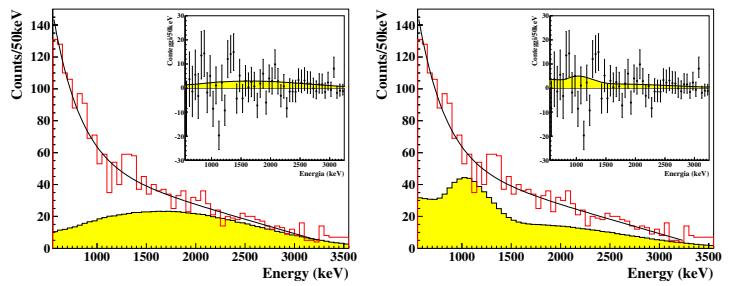

Fig. 2. Comparison between the experimental spectrum measured during $8823.54 \mathrm{~h}$ (thick histogram) and the expected signal (colored histogram) for: left) the $n p$ disappearance with $\tau_{n p}=4.0 \cdot 10^{22}$ yr excluded at $90 \%$ C.L.; right) the $p p$ disappearance with $\tau_{p p}=2.1 \cdot 10^{23} \mathrm{yr}$ excluded at $90 \%$ C.L. In the insets the residuals between the experimental spectrum and the background fits are shown together with excluded distributions for $n p\left(\tau_{n p}=3.2 \cdot 10^{23} \mathrm{yr}\right)$ and $p p\left(\tau_{p p}=1.9 \cdot 10^{24} \mathrm{yr}\right)$ disappearances, respectively. See text.

\subsection{Results for NN decays into invisible channel}

A possible $n p$ disappearance in the ${ }^{136} \mathrm{Xe}$ nucleus can be investigated by searching for the following $\beta^{-}$decay ${ }^{134} \mathrm{I} \rightarrow{ }^{134} \mathrm{Xe}\left(T_{1 / 2}=52.5 \mathrm{~m}, Q_{\beta^{-}}=4175 \mathrm{keV}\right)$ in the sensitive volume of DAMA/LXe. Considering the expected energy distribution calculated for this decay, the most sensitive energy region of the experimental spectrum (see fig. 2 left) is $2250-2300 \mathrm{keV}$ interval with 14 events collected: $S_{\Delta E}<18.8$ events at $90 \%$ C.L. With the related efficiency $\varepsilon_{\Delta E}=1.86 \%$ and the effective number of pairs $N_{n p}^{\text {eff }}=2$, we obtain the restriction on the $n p$ lifetime: $\tau_{n p}>4.0 \cdot 10^{22}$ yr $(90 \%$ C.L. $)$.

The result of a $p p$ disappearance in the ${ }^{136} \mathrm{Xe}$ nucleus is instead the creation of a ${ }^{134} \mathrm{Te}$ isotope and the subsequent $\beta^{-}$decays chain: ${ }^{134} \mathrm{Te} \rightarrow{ }^{134} \mathrm{I}\left(T_{1 / 2}=41.8 \mathrm{~m}, Q_{\beta^{-}}=\right.$ $1550 \mathrm{keV}$ ) and ${ }^{134} \mathrm{I} \rightarrow{ }^{134} \mathrm{Xe}$ (see above). The expected energy distribution for these processes in DAMA/LXe sensitive volume gives in particular for the ${ }^{134} \mathrm{Te} \beta^{-}$decay a bump at $\sim 1000 \mathrm{keV}$ due to events where the de-excitation $\gamma^{\prime}$ 's, following the $\beta^{-}$ray with end-point $\sim 730 \mathrm{keV}(42 \%$ of the cases), $\sim 610 \mathrm{keV}(44 \%)$ and $\sim 450 \mathrm{keV}(14 \%)$, are fully contained in the detector, and a structure with energy below $\sim 800 \mathrm{keV}$ associated with events where one or more $\gamma$ 's escape the detector. For the $p p$ disappearance the expected signal is given by the ${ }^{134} \mathrm{Te}+{ }^{134} \mathrm{I}$ distribution and the considered region of the experimental spectrum (see fig. 2 right) is $1100-1150 \mathrm{keV}$ with 35 events. With the $S_{\Delta E}<42.6, \varepsilon_{\Delta E}=6.47 \%$, and $N_{p p}^{\text {eff }}=7$, it results in the value $\tau_{p p}>2.1 \cdot 10^{23} \mathrm{yr}$ at $90 \%$ C.L.

However, when the experimental data have a smooth behaviour and the expected response function of the effect has some peculiarities, as the peak-like structure near $1 \mathrm{MeV}$ for the ${ }^{134} \mathrm{Te}+{ }^{134} \mathrm{I}$ decay ( $p p$ disappearance), it is justified to use an approach that allows to take into account the background contribution when estimating the limit on the lifetime. In particular, the experimental spectrum can be fitted by some appropriate background model together with the effect's response function with parameters of the model and the amplitude of the effect being the free parameters of the fit. The fit of the experimental 

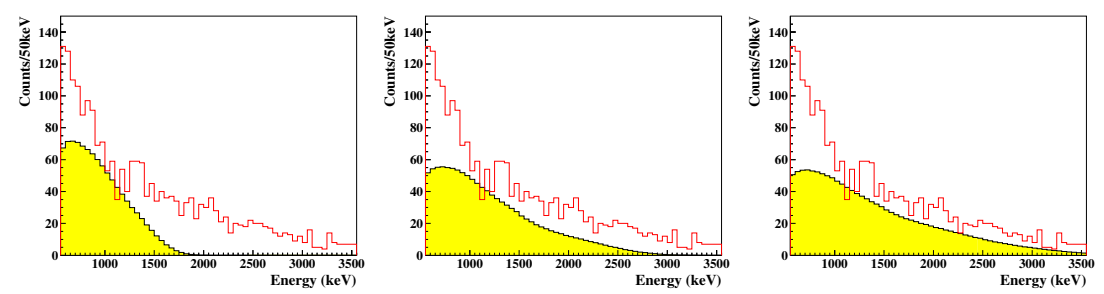

Fig. 3. Comparison between the experimental spectrum measured during $8823.54 \mathrm{~h}$ (thick histogram) and the expected signal (colored histogram) for: left) the $n n p$ disappearance with $\tau_{n n p}=1.4 \cdot 10^{22} \mathrm{yr}$ excluded at $90 \%$ C.L.; center) the $n p p$ disappearance with $\tau_{n p p}=2.7 \cdot 10^{22} \mathrm{yr}$ excluded at $90 \%$ C.L.; right) the $p p p$ disappearance with $\tau_{p p p}=3.6 \cdot 10^{22}$ yr excluded at 90\% C.L.

spectrum in the $550-3200 \mathrm{keV}$ region by the sum of an exponent and a straight line, as background model $^{2}$, and the ${ }^{134} \mathrm{Te}+{ }^{134} \mathrm{I}$ decay response function gave for its area the value $S=0.0 \pm 44.2$, providing no evidence for the effect being searched; the corresponding $\chi^{2} /$ n.d.f. value was equal to 1.1. According to the Feldman-Cousins procedure [37], the $90 \%$ C.L. limit on the number of observed ${ }^{134} \mathrm{Te}+{ }^{134} \mathrm{I}$ events is $S<72.5$. This value, together with the effective number of the $p p$ pairs $N_{p p}^{\text {eff }}=7$, results in the $p p$ lifetime limit:

$$
\tau_{p p}>1.9 \cdot 10^{24} \mathrm{yr} \text { at } 90 \% \text { C.L. }
$$

In the same way the limit on the number of events under the ${ }^{134} \mathrm{I}$ distribution ( $n p$ disappearance) was determined. The fit $\left(\chi^{2} /\right.$ n.d.f. $\left.=1.1\right)$ gave for the $S$ value $S=19.3 \pm 65.3$ that resulted in the bound $S<126.4$ at $90 \%$ C.L. Taking into account that $N_{n p}^{\text {eff }}=2$, we obtain the $n p$ lifetime limit:

$$
\tau_{n p}>3.2 \cdot 10^{23} \text { yr at } 90 \% \text { C.L. }
$$

The distributions corresponding to a lifetime equal to these limit values for the $n p$ and $p p$ disappearances in ${ }^{136} \mathrm{Xe}$ are shown together with the residuals between the experimental spectrum and the background model in the insets of fig. 2 left and right, respectively.

\subsection{Results for NNN decays into invisible channel}

The result of a nnn $\rightarrow$ invisible channels decay in the ${ }^{136} \mathrm{Xe}$ nucleus is the creation of the ${ }^{133} \mathrm{Xe}$ isotope, which is unstable and $\beta^{-}$decays to ${ }^{133} \mathrm{Cs}$ (stable) with $T_{1 / 2}=$ $5.243 \mathrm{~d}$ and $Q_{\beta^{-}}=427.4 \mathrm{keV}$. As evident, the maximum energy released in this process is below the $550 \mathrm{keV}$ energy threshold of the present measurements; thus, this decay process cannot be investigated here.

As a consequence of a $n n p$ disappearance in the ${ }^{136} \mathrm{Xe}$ nucleus, a ${ }^{133}$ I nucleus is instead created in the sensitive volume of DAMA/LXe. This isotope is the parent of the $\beta^{-}$decay chain ${ }^{133} \mathrm{I} \rightarrow{ }^{133} \mathrm{Xe} \rightarrow{ }^{133} \mathrm{Cs}$. The energy distribution expected for the ${ }^{133} \mathrm{I} \beta^{-}$decay $\left(T_{1 / 2}=20.8 \mathrm{~h}\right.$ and $Q_{\beta^{-}}=1770 \mathrm{keV}$ ) in our liquid xenon set-up is mainly determined by the $\beta^{-}$decay with end-point $\sim 1240 \mathrm{keV}$ ( $83 \%$ of the cases) followed by a $530 \mathrm{keV}$ de-excitation $\gamma$

${ }^{2}$ Other parameterizations gave similar results. (the $\beta^{-}$energy distribution is moved towards higher energy of $530 \mathrm{keV}$ ); a $233 \mathrm{keV}$ peak is instead due to the deexcitation of the ${ }^{133} \mathrm{Xe}$ metastable state $\left(T_{1 / 2}=2.19 \mathrm{~d}\right)$, which is reached by the $\sim 3 \%$ of the decays [11]. The signal to be searched for in case of a $n n p$ disappearance in ${ }^{136} \mathrm{Xe}$ is given by the ${ }^{133} \mathrm{I}+{ }^{133} \mathrm{Xe} \beta^{-}$decay distributions but the last one in the analysis does not play any role since it falls under the experimental energy threshold ( $550 \mathrm{keV})$.

The more selective region in the present case is $\Delta E=$ $1100-1150 \mathrm{keV}$ (see fig. 3 left); it contains 35 events, which gives rise to the upper limit (90\% C.L.) $S_{\Delta E}<42.6$ events and being $\epsilon_{\Delta E}=3.0 \%$ gives:

$$
\tau_{n n p}>1.4 \cdot 10^{22} \mathrm{yr} \text { at } 90 \% \text { C.L. }
$$

In the case of the process $n p p \rightarrow$ invisible channels in the ${ }^{136} \mathrm{Xe}$ nucleus, a ${ }^{133} \mathrm{Te}$ nucleus is created. This isotope $\left(T_{1 / 2}=12.5 \mathrm{~m}\right.$ and $\left.Q_{\beta^{-}}=2920 \mathrm{keV}\right)$ produces a $\beta^{-}$decay to ${ }^{133} \mathrm{I}$ followed by the decay chain ${ }^{133} \mathrm{I} \rightarrow{ }^{133} \mathrm{Xe} \rightarrow{ }^{133} \mathrm{Cs}$ already described above for the $n n p$ channel. The simulation result for a ${ }^{133}$ Te decay in the DAMA/LXe detector gives peculiar structures in the expected distribution determined by the $\gamma$ 's emitted in the ${ }^{133}$ I de-excitation, whose energies are summed to the initial $\beta^{-}$of ${ }^{133} \mathrm{Te}$. The more probable emitted $\gamma$ 's are those with energies $312 \mathrm{keV}(62 \%)$ and $408 \mathrm{keV}(27 \%)$, from the first two excited levels of ${ }^{133} \mathrm{I}$. In fact, a bump at $\sim 300 \mathrm{keV}$ is due to the $\gamma$ at $312 \mathrm{keV}$ which, in most cases, releases all its energy in the detector, while a bump at $\sim 700 \mathrm{keV}$ is due to the sum of the energies released by the two $\gamma$ 's emitted in cascade. Each possible $n p p$ decay in ${ }^{136} \mathrm{Xe}$ is associated to a signal given by the sum of the energy distributions expected for the $\beta^{-}$decays of the ${ }^{133} \mathrm{Te}$, of the ${ }^{133} \mathrm{I}$ and of the ${ }^{133} \mathrm{Xe}$ (this latter, as already mentioned, gives here a signal under the experimental energy threshold and, therefore, is not considered) [11]. Comparing the experimental spectrum measured (see fig. 3 center) and the expected signal, one gets that the more selective energy window is $\Delta E=1100-1150 \mathrm{keV}$ which contains 35 events, giving rise to the upper limit: $S_{\Delta E}<42.6$ events (90\% C.L.). Taking into account the calculated efficiency $\epsilon_{\Delta E}=5.7 \%$, one can derive

$$
\tau_{n p p}>2.7 \cdot 10^{22} \text { yr at } 90 \% \text { C.L. }
$$

Finally, a possible $p p p \rightarrow$ invisible channels decay in ${ }^{136} \mathrm{Xe}$ will create in the liquid xenon the ${ }^{133} \mathrm{Sb}$ nucleus which $\beta^{-}$decays with $T_{1 / 2}=2.5 \mathrm{~m}$ and $Q_{\beta^{-}}=4003 \mathrm{keV}$. As can be derived from the decay scheme of the ${ }^{133} \mathrm{Sb}$, this 


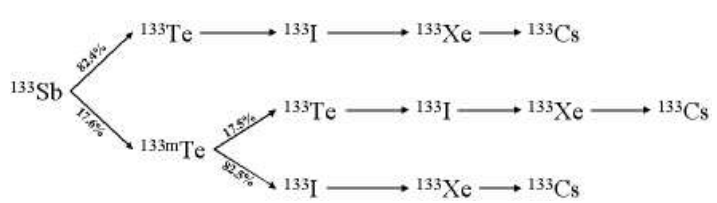

Fig. 4. The full decay chain generated by the ${ }^{133} \mathrm{Sb}$.

process gives rise to the production of ${ }^{133} \mathrm{Te}$ nuclei in the ground state $(82.4 \%)$ and in the ${ }^{133 \mathrm{~m}}$ Te metastable state at $334 \mathrm{keV}(17.6 \%)$. The full decay chain generated by the ${ }^{133} \mathrm{Sb}$ is summarized in fig. 4 . With simple calculation one can get that a possible $p p p$ disappearance in ${ }^{136} \mathrm{Xe}$ is followed by a sequence of $\beta^{-}$decays weighted according to the following formula:

$$
\begin{aligned}
& 1 \times{ }^{133} \mathrm{Sb}+0.855 \times{ }^{133} \mathrm{Te}+0.176 \times{ }^{133 \mathrm{~m}} \mathrm{Te} \\
& +1 \times{ }^{133} \mathrm{I}+1 \times{ }^{133} \mathrm{Xe} .
\end{aligned}
$$

The energy distributions expected for the ${ }^{133} \mathrm{Te},{ }^{133} \mathrm{I}$ and ${ }^{133} \mathrm{Xe}$ isotopes have been already described above. As regards the response function of DAMA/LXe for the $\beta^{-}$ decay of ${ }^{133} \mathrm{Sb}$, it is more difficult to identify the structure due to the $\gamma$ 's emitted in the daughter nucleus deexcitation. In fact, the more probable $\gamma$ 's have energies of the order of $\mathrm{MeV}$ and, therefore, they can escape the detector releasing only a part of their energy. The last process in $(8)$ is the ${ }^{133 \mathrm{~m}} \mathrm{Te}$ decay $\left(T_{1 / 2}=55.4 \mathrm{~m}\right)$. The expected energy distribution for the ${ }^{133 \mathrm{~m}}$ Te decay process gives a $334 \mathrm{keV}$ peak due to the de-excitation $\gamma$, which in the $17.5 \%$ of the cases move the ${ }^{133 \mathrm{~m}}$ Te into the ground state of ${ }^{133} \mathrm{Te}$; the remaining part of the expected signal (branching ratio $=82.5 \%$ ) is instead given by the ${ }^{133 \mathrm{~m}} \mathrm{Te}$ $\beta^{-}$decay $\left(Q_{\beta^{-}}=2920 \mathrm{keV}\right)$ to ${ }^{133} \mathrm{I}$. In particular, the presence of the ${ }^{133} \mathrm{I}$ metastable state at $1634 \mathrm{keV}$ with $T_{1 / 2}=9 \mathrm{~s}$ induces other peaks in the expected energy distribution (a $74 \mathrm{keV}$ peak which can be well distinguished, but also peaks at $721 \mathrm{keV}, 987 \mathrm{keV}$ and $1634 \mathrm{keV}$ ) [11]. The signal expected for the $p p p \rightarrow$ invisible channels in ${ }^{136} \mathrm{Xe}$ can be obtained by summing according to (8) the obtained energy distributions [11] (we remind that the signal associated to the ${ }^{133} \mathrm{Xe}$ decay is below the experimental threshold $550 \mathrm{keV})$. Considering the expected energy distribution (see fig. 3 right), the most sensitive energy window is $\Delta E=1100-1150 \mathrm{keV}$ which contains 35 events, giving rise to the upper limit: $S_{\Delta E}<42.6$ events (90\% C.L.); the detection efficiency is: $\epsilon_{\Delta E}=7.6 \%$. One obtains

$$
\tau_{p p p}>3.6 \cdot 10^{22} \text { yr at } 90 \% \text { C.L. }
$$

\section{Conclusion}

The DAMA/LXe set-up, deeply improved several times, has allowed to achieve competitive results in the search for various rare processes as summarized in the first section of this paper.

Here in particular, the most recent results on nucleon decays into invisible channels in the ${ }^{136} \mathrm{Xe}$ isotope have
Table 3. Experimental limits $(90 \%$ C.L.) obtained by DAMA/LXe on the lifetime values of $N, N N$ and $N N N$ decays into invisible channels in the ${ }^{136} \mathrm{Xe}$ isotope.

\begin{tabular}{|c|c|}
\hline Decay & $\tau_{\text {limit }}$ years $(90 \%$ C.L. $)$ \\
\hline \hline$n$ & $3.3 \cdot 10^{23}$ \\
\hline$p$ & $4.5 \cdot 10^{23}$ \\
\hline$n n$ & - \\
\hline$n p$ & $3.2 \cdot 10^{23}$ \\
\hline$p p$ & $1.9 \cdot 10^{24}$ \\
\hline$n n n$ & - \\
\hline$n n p$ & $1.4 \cdot 10^{22}$ \\
\hline$n p p$ & $2.7 \cdot 10^{22}$ \\
\hline$p p p$ & $3.6 \cdot 10^{22}$ \\
\hline
\end{tabular}

been discussed. The considered experimental approach assures a high detection efficiency and a branching ratio $\sim 1$ with respect to other different approaches that used very large mass installations to compensate for the much lower values for those quantities. In particular, $N N N$ decays into invisible channels have been investigated here for the first time. The obtained results are summarized in table 3. All the limits achieved here are valid for every invisible decay channel, including disappearance in extra-dimensions or decay into particles which weakly interact with matter.

Further data taking is foreseen.

The authors thank F. Vissani for useful discussions.

\section{References}

1. P. Belli et al., Nuovo Cimento C 19, 537 (1996).

2. P. Belli et al., Astropart. Phys. 5, 217 (1996).

3. P. Belli et al., Phys. Lett. B 387, 222 (1996); 389, 783 (1996) (E).

4. R. Bernabei et al., New J. Phys. 2, 15.1 (2000).

5. R. Bernabei et al., EPJdirect C 11, 1 (2001).

6. R. Bernabei et al., Phys. Lett. B 436, 379 (1998).

7. R. Bernabei et al., in Beyond the Desert 2003 (Springer, 2003) p. 365.

8. R. Bernabei et al., Nucl. Instrum. Methods A 482, 728 (2002).

9. R. Bernabei et al., Phys. Lett. B 527, 182 (2002).

10. R. Bernabei et al., Phys. Lett. B 546, 23 (2002).

11. F. Cappella, PhD Thesis, Università di Roma "Tor Vergata" (2005).

12. P. Belli et al., Nuovo Cimento A 103, 767 (1990).

13. P. Belli et al., Phys. Rev. D 61, 117301 (2000).

14. P. Belli et al., Phys. Lett. B 465, 315 (1999).

15. R. Bernabei et al., Phys. Lett. B 493, 12 (2000).

16. R. Bernabei et al., in Technique and Application of Xenon Detectors (World Scientific Publ., 2002) p. 50.

17. F. Simkovic, P. Domin, A. Faessler, hep-ph/0204278.

18. A. Staudt et al., Europhys. Lett. 13, 31 (1990).

19. E. Caurier et al., Nucl. Phys. A 654, 973 (1999).

20. G. Feinberg, M. Goldhaber, Proc. Natl. Acad. Sci. U.S.A. 45, 1301 (1959). 
21. J.N. Bahcall, Rev. Mod. Phys. 50, 881 (1978); Neutrino Astrophysics (Cambridge University Press, 1989) p. 359.

22. E.C.G. Stuckelberg, Helv. Phys. Acta 11, 225 (1938).

23. E.P. Wigner, Proc. Am. Philos. Soc. 93, 521 (1949).

24. P. Langacker, Phys. Rep. 71, 185 (1981).

25. C.E. Carlson, C.D. Carone, Phys. Lett. B 512, 121 (2001).

26. R.N. Mohapatra et al., Phys. Lett. B 491, 143 (2000).

27. K.S. Babu et al., Phys. Lett. B 570, 32 (2003).

28. F.J. Yndurain, Phys. Lett. B 256, 15 (1991);

N. Arkani-Hamed et al., Phys. Lett. B 429, 263 (1998);

N. Arkani-Hamed et al., Phys. Today 55, February issue, 35 (2002).
29. S.L. Dubovsky et al., Phys. Rev. D 62, 105011 (2000); S.L. Dubovsky et al., JHEP 08, 041 (2000); V.A. Rubakov, Phys. Usp. 44, 871 (2001).

30. S.L. Dubovsky, JHEP 01, 012 (2002).

31. R.B. Firestone, V.S. Shirley et al. (Editors), Table of Isotopes, 8th ed. (John Wiley \& Sons, New York, 1996).

32. C. Berger et al., Phys. Lett. B 269, 227 (1991).

33. W.R. Nelson et al., SLAC-Report-265, Stanford, 1985.

34. S. Rab, Nucl. Data Sheets 75, 491 (1995).

35. Yu.V. Sergeenkov, Nucl. Data Sheets 71, 557 (1994).

36. Yu.V. Sergeenkov et al., Nucl. Data Sheets 84, 115 (1998).

37. G.J. Feldman, R.D. Cousins, Phys. Rev. D 57, 3873 (1998). 